\title{
AVANCES EN LA IDENTIFICACIÓN DE OBSIDIANAS UTILIZADAS EN EL VALLE DE HUALFín (DEPTO. DE BelÉN, CATAMARCA, ARgEntina) DURANTE EL PERÍODO DE DESARROLLOS REGIONALES/INKA
}

\author{
Marina Cecilia Flores y Bárbara María Balesta ${ }^{1}$
}

\begin{abstract}
En este trabajo se presentan los resultados del estudio macroscópico y geoquímico realizado sobre 10 muestras de obsidiana procedentes de siete sitios arqueológicos de las localidades de Asampay y de La Ciénaga de Abajo (valle de Hualfín, Catamarca, Argentina), correspondientes cronológicamente al período de Desarrollos Regionales/Inka

(1000-1535 DC.). El estudio geoquímico de las muestras permitió identificar como lugar de procedencia de las mismas a las fuentes Ona, Cueros de Purulla/Chascón y Laguna Cavi; mientras el estudio macroscópico posibilitó vincular a la mayor parte de las obsidianas Cueros de Purulla/Chascón a la primera de estas fuentes. La obsidiana, en general, ha sido un recurso intensamente aprovechado y se encuentra principalmente relacionada con la manufactura de puntas de proyectil.
\end{abstract}

Palabras claves: estudio macroscópico - análisis geoquímico - artefactos líticos tallados - noroeste argentino

\begin{abstract}
In this paper we present the results of the macroscopic and geochemical analyses performed on 10 samples of obsidian from seven archaeological sites from Asampay and La Ciénaga de Abajo localities (Hualfin valley,

Catamarca, Argentine) chronologically corresponding to Regional

Development/Inka Period (1000-1535 AD). The geochemical studies

of samples allowed identify the provenience source as Ona, Cueros

de Purulla/Chascón y Laguna Cavi; while macroscopic study enabled linking most of obsidians Cueros de Purulla/Chascón to the first of these sources. Obsidian, in general, has been an intensively exploited resource and is mainly related to the manufacture of projectile points.
\end{abstract}

Key words: macroscopic study - geochemical analyses - chipped stone tools - Northwest Argentina

Recibido: noviembre 2013. Aceptado: junio 2014.

\section{* Introducción}

En el noroeste argentino, los análisis de procedencia de obsidiana se iniciaron en 1989, profundizándose en la década de 1990, con la localización y caracterización tanto de muestras naturales, tomadas en diversos afloramientos como de artefactos arqueológicos, recuperados en distintos sitios de la región (Escola et al. 1992-1993; Escola 2000). La continuación de esta clase de estudios ha permitido que en la actualidad se conozcan tanto nuevas fuentes de obsidianas, como la procedencia de este tipo de materia prima presente en numerosos sitios adscriptos a distintos momentos temporales, vinculados a grupos humanos de diferente complejidad social y organización económica (Scatolín y Lazzari 1997; Escola 2000; Yacobaccio et al. 2004; Escola y Hocsman 2007; Sprovieri y Baldini 2007; Sprovieri y Glascock 2007; Elías 2008; Chaparro 2009; De Feo y Álvarez Soncini 2010; Elías 2011, entre otros). Se conocen a la fecha los siguientes afloramientos de obsidiana localizados en el noroeste argentino: Quirón, Ramadas, Alto Tocomar (Salta), Ona/Las Cuevas, Valle Ancho, Chascón, Cueros de Purulla, Laguna Cavi -antiguamente denominada "fuente desconocida B"- y Salar del Hombre Muerto -antiguamente denominada "fuente desconocida A"- (Catamarca); Caldera Vilama 1 y 2 (Jujuy) (Yacobaccio et al. 2004; Elías et al. 2009; Escola et al. 2009; Elías y Escola 2010). Por otra parte, se ha identificado obsidiana procedente de la fuente Zapaleri (Bolivia) en varios sitios arqueológicos del sector septentrional del noroeste argentino -provincias de Jujuy y

\footnotetext{
1 UNLP. Laboratorio de Análisis Cerámico. Facultad de Ciencias Naturales y Museo. Universidad Nacional de La Plata. marin481@hotmail.com y bbalesta@ciudad.com.ar
} 
Salta- (Yacobaccio et al. 2004; Escola 2007; Sprovieri y Glascock 2007). Es importante señalar, que en varios sitios de la provincia de Catamarca, y en menor medida de la Quebrada de Humahuaca, se recuperaron distintos tipos de obsidianas, correspondientes a fuentes aún desconocidas C, E, F, G, H, J, K, My N (Yacobaccio et al. 2004; Elías y Escola 2010).

En el valle de Hualfín (Depto. de Belén, Catamarca) esta clase de estudios se inició en el año 2008, cuando se enviaron al Missouri University Research Reactor (MURR) siete muestras correspondientes a desechos de talla de obsidiana hallados en dos sitios, denominados Loma de los Antiguos y Campo de Carrizal, ubicados en la localidad de Asampay y con ocupaciones estimadas principalmente entre la segunda mitad del siglo XV y el siglo XVI. La selección de las muestras estuvo basada en la observación de distintas características macroscópicas (color, transparencia y presencia de inclusiones); los resultados señalaron a la fuente de obsidiana Ona como lugar único de procedencia de todas las piezas analizadas, por lo cual se interpretó que las diferencias registradas a nivel macroscópico correspondían a la variación interna de la misma fuente (Flores y Morosi 2010).

El objetivo del presente trabajo consiste en avanzar en la identificación, caracterización y procedencia de las obsidianas utilizadas por los pobladores del valle de Hualfín durante el período de Desarrollos Regionales/Inka (1000-1535 DC.). En esta oportunidad se dan a conocer los resultados de los estudios macroscópicos y geoquímicos realizados sobre 10 artefactos líticos tallados recuperados en siete sitios arqueológicos de las localidades de Asampay y de La Ciénaga de Abajo. Los mismos permitieron aumentar y complementar la información existente sobre la procedencia de las obsidianas utilizadas en el valle para esos momentos, señalar la amplia distribución espacial de la obsidiana Ona dentro del área de estudio, así como el acceso irrestricto a este recurso, y además, identificar otras fuentes de obsidianas utilizadas paralelamente.

\section{El área de estudio}

El valle de Hualfín (Figura 1) corresponde a la región arqueológica Valliserrana del noroeste argentino (González y Pérez 1972). Se extiende desde la localidad de
Las Juntas hasta la denominada Puerta de Belén y se encuentra limitado al sureste por la Sierra de Belén, al noreste por las estribaciones de la Sierra de La Ovejería y al norte por la Sierra de Hualfín, mientras que por el oeste, el Cordón de Fiambalá lo separa del valle de Abaucán (González 1998).

El río Hualfín (también denominado Belén), que recorre este valle, nace en la confluencia de los ríos Nacimientos y Durazno, y desde allí inicia su recorrido con rumbo noreste-sudoeste, recibiendo el aporte de diversos ríos tributarios como el Loconte, Villavil, Corral Quemado, Agua Clara, entre otros. Durante su recorrido, el Hualfín arrastra gran cantidad de sedimento arenoso, proveniente mayoritariamente de la erosión de los estratos terciarios. El área corresponde a las Sierras Pampeanas Noroccidentales. Se trata de un basamento cristalino (PrecámbricoCámbrico) compuesto por rocas metamórficas variadas intruidas por granitoides. Sobre esta unidad se apoyan en discordancia sedimentitas neógenas, culminando la secuencia con depósitos de acarreo de edad cuaternaria (González Bonorino 1950, 1972; Ruíz Huidobro 1975).

El clima de la región es subtropical, con características semiáridas. Las temperaturas oscilan entre $9^{\circ} \mathrm{C}$ y $25^{\circ} \mathrm{C}$,

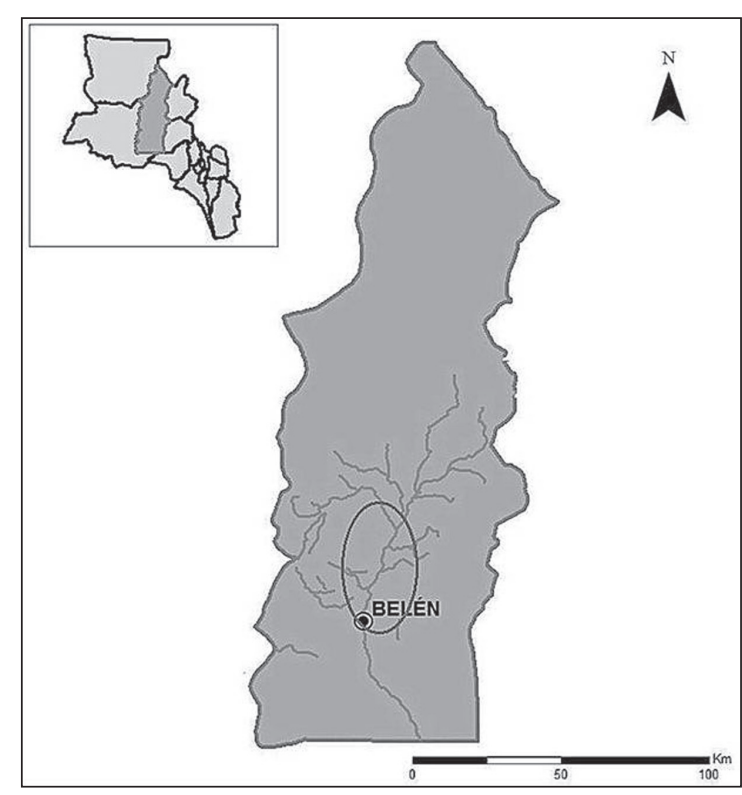

Figura 1. Ubicación espacial del valle de Hualfín dentro del Departamento de Belén, Catamarca, Argentina. 
con una media anual de $18^{\circ} \mathrm{C}$. En verano se producen marcadas diferencias de temperatura que afectan el régimen de los vientos, haciendo variar sus direcciones, forzándolos a subir por las laderas orientales, lo cual origina una pérdida de su humedad. Es por ello que la mayor cantidad de precipitaciones se contabiliza entre los $1000 \mathrm{y}$ $1500 \mathrm{msnm}$; mientras que a mayores alturas se producen lloviznas y neblinas persistentes y en alturas superiores a los $2500 \mathrm{msnm}$ se registran granizo, nieve y garrotillo (aguanieve). En invierno aumenta la influencia de los vientos secos, que van perdiendo su humedad a medida que se desplazan hacia el noreste. Por ello, el régimen pluviométrico exhibe una marcada variación estacional; la mayor cantidad de lluvias se registra durante el verano -precipitaciones entre 200 y $400 \mathrm{~mm}$ - y prácticamente no llueve durante el resto del año; el clima se presenta cálido en verano y frío en invierno; en primavera prepondera el viento zonda.

\section{El período de Desarrollos Regionales/Inka}

En el noroeste argentino, el período de Desarrollos Regionales se caracterizó por la conformación de organizaciones políticas más complejas que en períodos anteriores, por un aumento demográfico y la concentración de las poblaciones, así como por el incremento del desarrollo agrícola a través de la implementación de sistemas de irrigación artificial y del uso intensivo de la tierra para la explotación agrícola (Núñez Regueiro 1974).

Tarragó (2000) plantea para esta época dos aspectos importantes: un notable crecimiento demográfico y la aparición de sociedades pujantes, que poseían territorios bien controlados y defendidos desde los pukara, poblados defensivos en lomas y/o amurallados.

Las nuevas formas económicas y políticas, desarrolladas a partir del colapso del viejo orden religioso-político (Tarragó 2000) y, quizás también, una intensa sequía (Nielsen 2002) habrían desencadenado la competencia por recursos -pastos, aguas, animales, etc.- entre diferentes sociedades. Este estado general de conflicto endémico (Nielsen 2002, 2007a), dado en los Andes centrales y meridionales, habría influenciado los modos de vida de las sociedades y habría favorecido la consolidación de formaciones políticas multicomunitarias. Estos cambios se verían reflejados en el tamaño, emplazamiento y estruc- tura interna de los asentamientos residenciales, como ya se ha mencionado.

Asimismo, para este momento, Tarragó (2000) refiere a la existencia de un activo tráfico regional a corta y larga distancia, que articulaba todo los Andes meridionales y la presencia de colonias efectivas en los sectores de Puna y Yungas, dependientes de las sociedades asentadas en los valles.

Hacia mediados del siglo XV los Inkas habrían arribado al área, en momentos de la expansión del imperio desarrollada por el décimo gobernante. Núñez Regueiro (1974) da inicio, con dicha expansión, al período Imperial, caracterizado por la conquista de tierras y la instalación de nuevos tipos de asentamientos -tambos, centros administrativos y militares, pukaras- a partir de nuevas construcciones o de la modificación de las preexistentes.

En el valle de Hualfín, el período de Desarrollos Regionales se manifestó a través de grupos "Belén" conocidos sobre todo a través de su cerámica Belén Negro sobre Rojo. Sempé (1981, 1999, 2005) caracteriza a estos grupos como señoríos o cacicazgos asentados en el valle de Hualfín -núcleo geopolítico-, que con el tiempo se habrían dispersado hacia valles aledaños (valle de Abaucán), así como a otros ambientes más altos (Puna), generando situaciones de conquista u ocupación, mientras que en otras zonas encontraron resistencia (valle de Santa María). Al respecto, Tarragó (2000) menciona que los grupos Belén establecieron satélites en el valle de Abaucán (Departamento de Tinogasta, Catamarca), complementaron productos de altura con La Alumbrera (Departamento de Antofagasta de la Sierra, Catamarca) y productos provenientes de valles más bajos (Tucumán), a través de Andalgalá (Departamento de Andalgalá, Catamarca).

Las evidencias de la presencia efectiva del Tawantinsuyu en la zona de estudio se encuentran conformadas por sitios arqueológicos tales como, Pukara de Hualfín, Quillay y El Shincal y por la recuperación de restos materiales de filiación incaica junto con piezas del Tardío, asociados en contextos funerarios. Sin embargo, en los sitios analizados y considerados aquí no se han registrado evidencias directas de esta ocupación (Wynveldt 2009), aunque los 
fechados radiométricos, en general, abarcan rangos de ocupación calibrados entre los siglos XIV y XVII.

Dentro del valle, los sitios correspondientes a este lapso temporal presentan distinta modalidad de emplazamiento. Se registran poblados sobre lomadas de más de 100 $\mathrm{m}$ de altura, con pendientes acentuadas que dificultan su acceso, los cuales se encuentran en muchos casos, además, protegidos por murallas defensivas, p. e. El Molino y Loma de la Escuela Vieja (Puerta de Corral Quemado), Cerrito Colorado (La Ciénaga de Arriba), entre otros, además de dos de los sitios incluidos en este trabajo: Loma de los Antiguos (Asampay) y Cerro Colorado (La Ciénaga de Abajo). Otros sitios, en cambio, se emplazaron entre campos de cultivos y estuvieron asociados a obras hidráulicas (acequias y estanques), p. e. Campo de Carrizal (Asampay). Por último, unos pocos se ubicaron en las terrazas de los ríos principales, p. e. Lajas Rojas, Barrancas, Barranca sur y Loma de Ichanga (La Ciénaga de Abajo).

\section{* Los Sitios y los Materiales considerados}

Como ya se ha reseñado, las muestras de artefactos líticos tallados en obsidiana que se estudian en este trabajo fueron recuperadas en siete sitios arqueológicos. Dos de ellos se encuentran en la localidad de Asampay, Loma de los Antiguos y Campo de Carrizal, mientras que los cinco restantes están en la localidad de La Ciénaga de Abajo: Cerro Colorado de La Ciénaga de Abajo, Lajas Rojas 2 y 4, Barrancas y Loma de Ichanga (Figura 2).

Los sitios presentan diferencias entre sí, principalmente en cuanto a su ubicación topográfica dentro del valle, cantidad y concentración de recintos y presencia o ausencia de murallas defensivas como se ha mencionado. A partir de las excavaciones realizadas en ellos se han recuperado distintos tipos de restos materiales, tales como fragmentos cerámicos ordinarios y decorados -fundamentalmente cerámica Belén Negro sobre Rojo-, restos vegetales (mayoritariamente carbonizados), restos óseos humanos

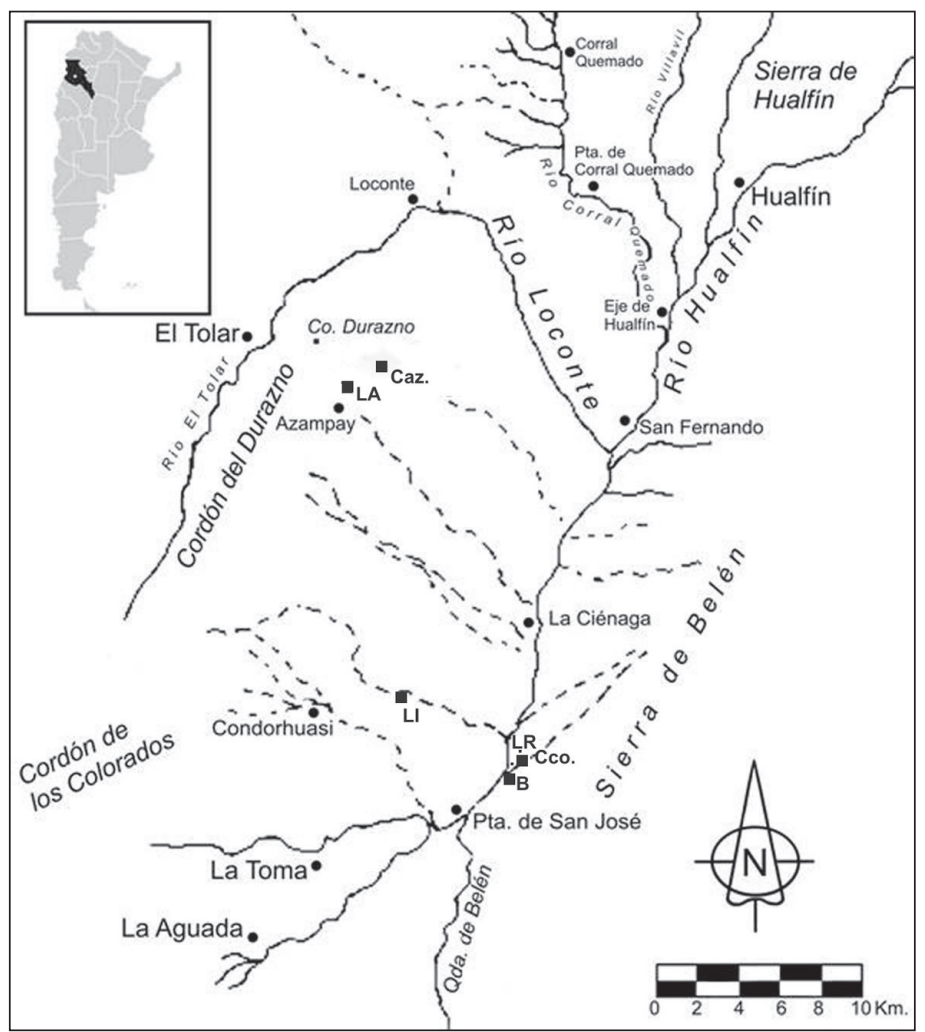

Figura 2. Ubicación de los sitios considerados dentro del valle de Hualfín (Depto. de Belén, Catamarca, Argentina). 


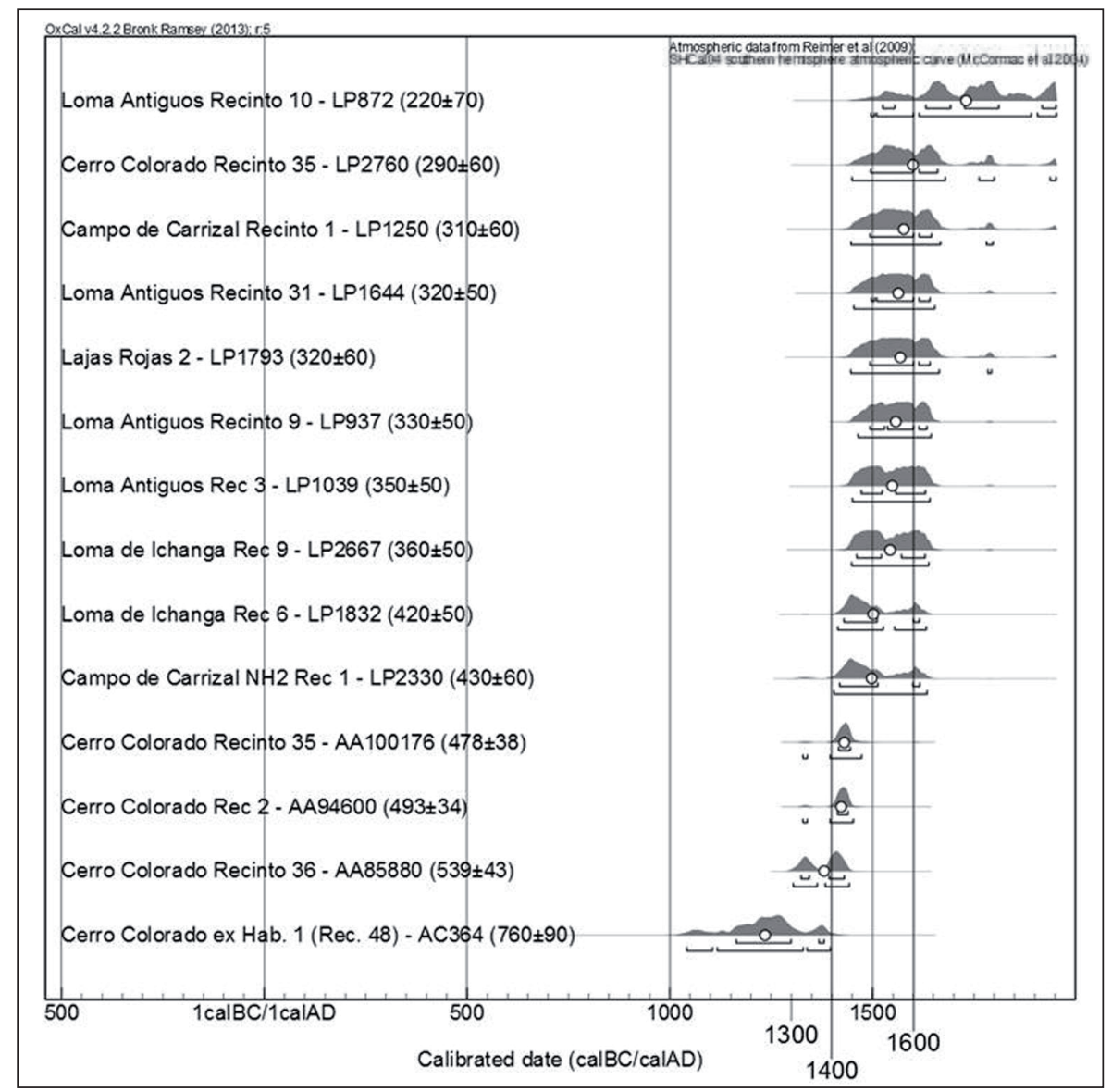

Figura 3. Fechados radiocarbónicos calibrados de los sitios considerados, realizados desde la década de 1990 (gentileza del Dr. F. Wynveldt).

y faunísticos, artefactos líticos tallados y pulidos, artefactos líticos de molienda, entre otros. Los fechados radiocarbónicos obtenidos para los mismos, junto con la presencia de cerámica Belén Negro sobre Rojo y las características constructivas observadas permiten plantear la ocupación de los sitios en general durante el período de Desarrollos Regionales/Inka (Figura 3). ${ }^{2}$

Los conjuntos de artefactos líticos tallados, recuperados en superficie y/o excavación en los diferentes sitios mencionados, están representados tanto por materias primas

2 Algunos sitios presentan fechados que extienden su ocupación hasta momentos del período Hispano-indígena. Sin embargo, no se encontraron evidencias materiales vinculadas con esos momentos. locales como no locales, entre éstas últimas se encuentra comprendida la obsidiana, objeto de nuestro trabajo. En la Tabla 1 y la Figura 4 se presentan, en forma resumida, los datos mencionados, sitio por sitio.

\section{* Metodología}

En primer lugar se procedió a la selección de muestras de obsidianas de cada uno de los siete sitios considerados. La misma se realizó en base a criterios macroscópicos tales como el color, la transparencia y la presencia o no de inclusiones. En el caso de los dos sitios de la localidad de Asampay se tuvieron en cuenta únicamente piezas que, en un primer estudio, no fueron identificadas macroscópicamente como obsidianas (Flores y Morosi 2010). A 


\begin{tabular}{|c|c|c|c|c|c|c|}
\hline \multicolumn{5}{|c|}{ Materias primas } & \multicolumn{3}{c|}{ Obsidianas } \\
\cline { 1 - 3 } Locales & $\%$ & N & $\%$ & N & \% del conjunto & $\%$ no locales \\
\hline 33 & 18 & 151 & 82 & 144 & 78 & 95 \\
\hline 246 & 64 & 139 & 36 & 116 & 30 & 83 \\
\hline 67 & 85 & 12 & 15 & 12 & 15 & 100 \\
\hline 1 & 33 & 2 & 67 & 2 & 67 & 100 \\
\hline 1 & 25 & 3 & 75 & 3 & 75 & 100 \\
\hline 3 & 60 & 2 & 40 & 2 & 40 & 100 \\
\hline 10 & 77 & 3 & 23 & 3 & 23 & 100 \\
\hline
\end{tabular}

Tabla 1. Artefactos líticos tallados de excavación y/o superficie de los sitios considerados y materias primas locales y no locales. Referencias: LA (Loma de los Antiguos), Caz (Campo de Carrizal), Cco (Cerro Colorado), LR2 (Lajas Rojas 2), LR4 (Lajas Rojas 4), B (Barrancas) y LI (Loma de Ichanga).

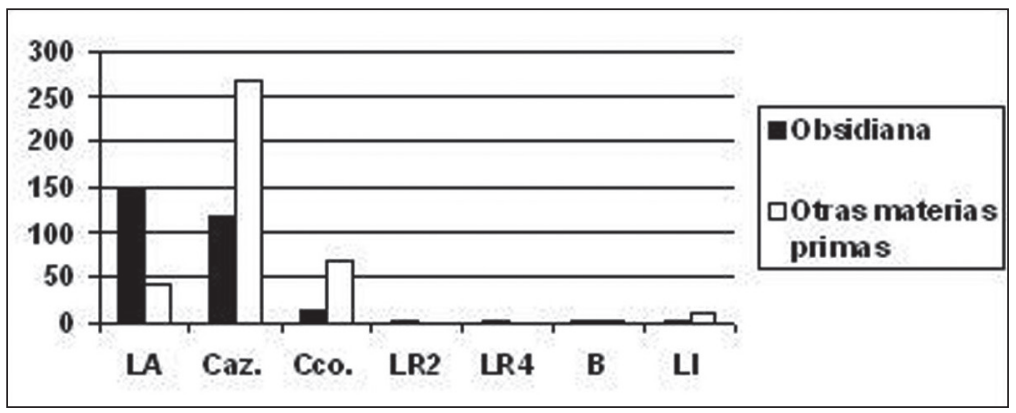

Figura 4. Presencia de obsidianas en comparación con otras materias primas por sitio. LA (Loma de los Antiguos), Caz (Campo de Carrizal), Cco (Cerro Colorado), LR2 (Lajas Rojas 2), LR4 (Lajas Rojas 4), B (Barrancas) y LI (Loma de Ichanga).

partir de tal segmentación se diferenciaron cinco grupos y se escogió un total 10 muestras para estudiar. El criterio consistió en tener al menos un ejemplar analizado por sitio y atender a las variaciones, dentro de un mismo grupo, que correspondían a piezas procedentes de diferentes sitios.

El conjunto a analizar $(\mathrm{N}=10$; Figura 5) quedó conformado por dos muestras del sitio Loma de los Antiguos (LA-156 y LA-157), cuatro muestras de Campo de Carrizal (CCA-471, CCA-563, CCA-564 y CDARS-1), una muestra de Cerro Colorado (CCRS-6), una muestra de Lajas Rojas 2 (LR-1), una muestra de Barrancas (B1-1) y una muestra de Loma de Ichanga (LI-1).

Las muestras elegidas fueron comparadas a nivel macroscópico con muestras arqueológicas y naturales de obsidianas, cuyas fuentes potenciales de aprovisionamiento fueron conocidas a partir de la realización de estudios previos (Flores y Morosi 2010) y de la evidencia aportada por la Dra. Elías (muestras de mano de obsidianas de las fuentes Ona, Cueros de Purulla, Chascón, Laguna Cavi, Ramadas, Alto Tocomar y Quirón). La comparación preliminar permitió reconocer similitudes entre algunas de ellas, por lo cual se decidió enviarlas para su análisis, a fin corroborar o no su vinculación. En la Tabla 2 se presenta la caracterización de las muestras seleccionadas.

En una siguiente etapa, las muestras seleccionadas fueron enviadas al Missouri University Research Reactor (MURR), en donde el Dr. Glascock llevó a cabo los análisis geoquímicos, por fluorescencia de rayos $\mathrm{x}$ (FRX). A partir de los mismos se determinaron 11 elementos traza: 


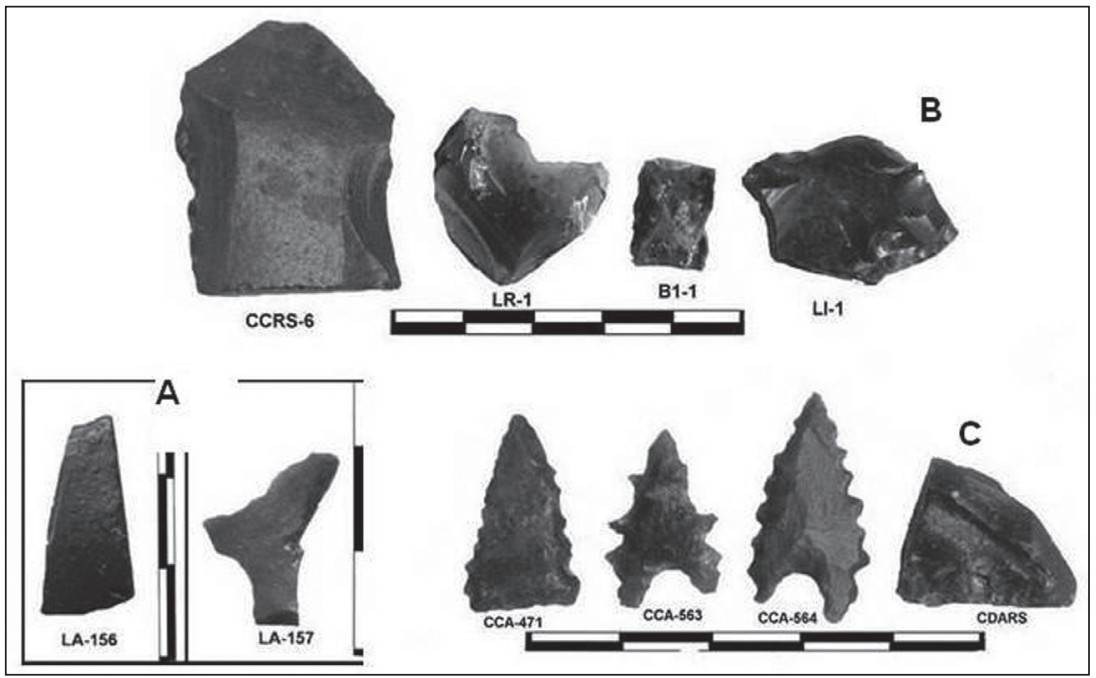

Figura 5. Muestras de obsidianas enviadas al MURR: A) Loma de los Antiguos; B) Diferentes sitios: Cerro Colorado, Lajas Rojas, Barrancas y Loma de Ichanga y C) Campo de Carrizal.

\begin{tabular}{|c|c|c|c|}
\hline Muestra & Descripción & Procedencia & $\begin{array}{c}\text { Semejanzas macroscópicas con obsi- } \\
\text { dianas de fuentes identificadas }\end{array}$ \\
\hline LA-156 & $\begin{array}{l}\text { lasca plana entera de } 23 \mathrm{~mm} \text { de longitud, } \\
26 \mathrm{~mm} \text { de ancho y } 3 \mathrm{~mm} \text { de espesor, con } \\
\text { talón facetado y terminación aguda }\end{array}$ & recinto $n^{\circ} 3$ (superficie) & No \\
\hline LA-157 & $\begin{array}{l}\text { lasca angular entera de } 17 \mathrm{~mm} \text { de longi- } \\
\text { tud, } 20 \mathrm{~mm} \text { de ancho y } 3 \mathrm{~mm} \text { de espesor, } \\
\text { con talón facetado y terminación aguda }\end{array}$ & recinto $n^{\circ} 3$ (superficie) & obsidiana Cueros de Purulla (variedad 2a) \\
\hline CCA-471 & $\begin{array}{l}\text { punta de proyectil apedunculada de } 21 \\
\mathrm{~mm} \text { de longitud, } 12 \mathrm{~mm} \text { de ancho y } 3 \mathrm{~mm} \\
\text { de espesor }\end{array}$ & $\begin{array}{l}\text { recinto } n^{\circ} 2 \text { del Núcleo habitacional } 1 \text { del } \\
2^{\circ} \text { espolón (excavación) }\end{array}$ & $\begin{array}{l}\text { obsidianas de las fuentes Cueros de } \\
\text { Purulla (variedad 2b) y Ramadas }\end{array}$ \\
\hline $\mathrm{CCA}-563$ & $\begin{array}{l}\text { punta de proyectil apedunculada de } 19 \\
\mathrm{~mm} \text { de longitud, } 12 \mathrm{~mm} \text { de ancho y } 3 \mathrm{~mm} \\
\text { de espesor }\end{array}$ & $\begin{array}{l}\text { recinto } n^{\circ} 2 \text { del Núcleo habitacional } 1 \text { del } \\
2^{\circ} \text { espolón (excavación). }\end{array}$ & obsidiana Laguna Cavi \\
\hline CCA-564 & $\begin{array}{l}\text { punta de proyectil apedunculada de } 25 \\
\text { mm de longitud, } 13 \mathrm{~mm} \text { de ancho y } 3 \mathrm{~mm} \\
\text { de espesor, }\end{array}$ & $\begin{array}{l}\text { recinto } n^{\circ} 2 \text { del Núcleo habitacional } 1 \text { del } \\
2^{\circ} \text { espolón (excavación) }\end{array}$ & obsidiana Cueros de Purulla (variedad 2a) \\
\hline CDARS-1 & $\begin{array}{l}\text { lasca plana entera de } 22 \mathrm{~mm} \text { de longitud, } \\
29 \mathrm{~mm} \text { de ancho y } 10 \mathrm{~mm} \text { de espesor, con } \\
\text { talón facetado y terminación en charnela }\end{array}$ & en el $2^{\circ}$ espolón (superficial) & obsidiana Laguna Cavi \\
\hline CCRS- 6 & $\begin{array}{l}\text { lasca angular entera de } 60 \mathrm{~mm} \text { de longi- } \\
\text { tud, } 40 \mathrm{~mm} \text { de ancho y } 14 \mathrm{~mm} \text { de espesor, } \\
\text { talón liso y terminación aguda }\end{array}$ & $\begin{array}{l}\text { pared noroeste del recinto } n^{\circ} 36 \text { (super- } \\
\text { ficie) }\end{array}$ & obsidiana Cueros de Purulla (variedad 2a) \\
\hline LR-1 & $\begin{array}{l}\text { lasca angular entera con talón astillado } \\
\text { y terminación en charnela, que presenta } \\
24 \mathrm{~mm} \text { de longitud, } 24 \mathrm{~mm} \text { de ancho y } 6 \\
\mathrm{~mm} \text { de espesor }\end{array}$ & recinto n² (excavación) & obsidiana Ona \\
\hline $\mathrm{B} 1-1$ & $\begin{array}{l}\text { desecho indiferenciado de } 15 \mathrm{~mm} \text { de lon- } \\
\text { gitud, } 11 \mathrm{~mm} \text { de ancho y } 7 \mathrm{~mm} \text { de espesor }\end{array}$ & estructura n³ (excavación) & obsidiana de la fuente Ona \\
\hline LI-1 & $\begin{array}{l}\text { lasca angular entera con talón facetado } \\
\text { y terminación en charnela, de } 16 \mathrm{~mm} \text { de } \\
\text { longitud, } 32 \mathrm{~mm} \text { de ancho y } 10 \mathrm{~mm} \text { de } \\
\text { espesor }\end{array}$ & cercanías del recinto $n^{\circ} 9$ (superficie) & obsidiana Ona \\
\hline
\end{tabular}

Tabla 2. Características de las muestras estudiadas. 
Mn, Fe, Zn, Ga, Rb, Sr, Y, Zr, Nb, Pb y Th. Sin embargo, algunos de esos elementos no fueron utilizados en la determinación, dado que los artefactos analizados resultaron demasiado pequeños, recayendo el análisis principalmente sobre cinco de ellos -Rb, Sr, Y, Zr y Nb- (Glascock, comunicación personal). Los resultados obtenidos fueron comparados con una base de datos que posee el mismo laboratorio, sobre fuentes de obsidiana identificadas en el noroeste argentino, con el objeto de localizar el o los lugares de proveniencia de las muestras analizadas.

Por último, y a partir de los resultados de los análisis macroscópicos y geoquímicos, se procedió a la identificación y segmentación de los conjuntos de obsidiana de cada uno de los sitios analizados, con la finalidad, por un lado de reconocer las fuentes de procedencia vinculadas con los mismos y por otro, su frecuencia.

\section{* Presentación de los resultados}

\section{Análisis geoquímico y procedencia de las muestras}

A partir de los estudios geoquímicos realizados, las muestras fueron asignadas a las fuentes Ona $(\mathrm{N}=3)$, Cueros de Purulla/Chascón $(\mathrm{N}=5)$ y Laguna Cavi $(\mathrm{N}$ = 2) (Tabla 3 y Figura 6). La mayor cantidad de muestras (LA-156, LA-157, CCA-471, CCA-574 y CCRS-6) fueron atribuidas a dos fuentes potenciales de aprovisionamiento espacialmente muy cercanas entre sí: Cueros de Purulla y Chascón. La diferenciación entre estas fuentes no pudo realizarse a través de fluorescencia de rayos $\mathrm{X}$ (FRX), siendo probable que correspondan a un mismo magma volcánico y difieran sólo ligeramente entre sí (Glascock, comunicación personal).

Los estudios de procedencia realizados hasta el momento permiten plantear entonces la vinculación entre diferentes fuentes de obsidianas localizadas dentro de la provincia de Catamarca y el valle de Hualfín (Figura 7). Estas fuentes se describen brevemente a continuación, siguiendo lo planteado en Escola (2004), Yacobaccio et al. (2004) y Escola et al. (2009).

La fuente Ona se encuentra a $3700 \mathrm{msnm}$, a aproximadamente $80-90 \mathrm{~km}$ de la localidad de Antofagasta de la Sierra y se compone de varios afloramientos y sectores de gran densidad de nódulos. En las adyacencias de la fuente también se presentan rodados de obsidiana, que habrían sido desplazados por la acción de la gravedad y otros procesos geológicos. Este vidrio volcánico traslúcido se presenta mayoritariamente en color negro y gris-negro, aunque se lo puede encontrar en otros colores, tales como gris claro, plateado y marrón rojizo; es de buena calidad para la talla y aparece bajo la forma de nódulos de hasta $20-30 \mathrm{~cm}$ de diámetro.

La fuente de obsidiana Cueros de Purulla se encuentra a $4380 \mathrm{msnm}$, aproximadamente a $60-70 \mathrm{~km}$ al sureste de la localidad de Antofagasta de la Sierra y presenta excelente calidad para la talla. La misma se manifiesta bajo la forma de grandes bloques y nódulos de hasta $25 \mathrm{~cm}$ de altura y es opaca, presentándose en varios colores tales como gris oscuro, negro rojizo y negro, siendo este último el más frecuente.

También a unos 60-70 km de Antofagasta de la Sierra se localiza la fuente Chascón, a $4290 \mathrm{msnm}$. La obsidiana se presenta bajo la forma de nódulos de color negro traslúcido, de más de $10 \mathrm{~cm}$ de diámetro; se trata de un vitrófiro con abundantes fenocristales.

Por último, la fuente Laguna Cavi está situada al sursuroeste del volcán Galán. Se trata de un depósito secundario de obsidiana integrado por nódulos de tamaño pequeño. La obsidiana aparece dispersa en los sedimentos de los paleocostas y paleoabanicos del extremo sureste de la laguna. Se trata de un material semi traslúcido de color gris oscuro. Escola y colaboradores (2009) señalan que los análisis geoquímicos realizados sobre muestras de esta materia prima mostraron una gran coincidencia con la denominada "fuente desconocida B".

\section{Resultados de la comparación macroscópica y geoquímica}

Es interesante señalar que a partir de los resultados de la comparación macroscópica realizada entre las muestras arqueológicas bajo estudio y las muestras arqueológicas y naturales correspondientes a fuentes identificadas, fue posible observar la correspondencia general previamente establecida y, además, plantear que las muestras asignadas a las fuentes Cueros de Purulla/Chascón corresponden 


\begin{tabular}{|c|c|c|c|c|c|c|c|c|c|c|c|c|}
\hline Muestra & Mn & $\mathrm{Fe}$ & $\mathrm{Zn}$ & $\mathrm{Ga}$ & $\mathrm{Rb}$ & Sr & $\mathbf{Y}$ & $\mathrm{Zr}$ & $\mathrm{Nb}$ & $\mathrm{Pb}$ & Th & Fuente \\
\hline LA-156 & 163 & 21404 & 100 & 16 & 155 & 259 & 13 & 173 & 8 & 30 & 14 & $\mathrm{CP} / \mathrm{CH}$ \\
\hline LA-157 & 364 & 9500 & 41 & 15 & 159 & 335 & 20 & 147 & 23 & 29 & 17 & $\mathrm{CP} / \mathrm{CH}$ \\
\hline CCA-471 & 329 & 9725 & 48 & 16 & 162 & 302 & 24 & 158 & 29 & 32 & 14 & $\mathrm{CP} / \mathrm{CH}$ \\
\hline CCA- 563 & 491 & 4622 & 40 & 18 & 484 & 10 & 59 & 54 & 37 & 40 & 33 & LC \\
\hline CCA-564 & 444 & 10611 & 59 & 16 & 172 & 308 & 23 & 160 & 33 & 29 & 8 & $\mathrm{CP} / \mathrm{CH}$ \\
\hline CDARS-1 & 571 & 3902 & 34 & 17 & 426 & 12 & 45 & 51 & 33 & 32 & 35 & LC \\
\hline CCRS-6 & 433 & 8560 & 48 & 16 & 175 & 369 & 26 & 159 & 29 & 27 & 22 & $\mathrm{CP} / \mathrm{CH}$ \\
\hline LR-1 & 260 & 5353 & 30 & 15 & 219 & 170 & 13 & 90 & 16 & 32 & 24 & $\mathrm{O}$ \\
\hline B1-1 & 361 & 5081 & 26 & 15 & 219 & 171 & 18 & 88 & 15 & 27 & 22 & $\mathrm{O}$ \\
\hline LI-1 & 507 & 5020 & 30 & 15 & 218 & 177 & 12 & 92 & 18 & 30 & 22 & $\mathrm{O}$ \\
\hline
\end{tabular}

Tabla 3. Detalle de los elementos traza pertenecientes a las muestras de obsidiana enviadas al MURR. Referencia de las fuentes: $\mathrm{CP} / \mathrm{CH}$ (Cueros de Purulla/Chascón), LC (Laguna Cavi) y O (Ona).

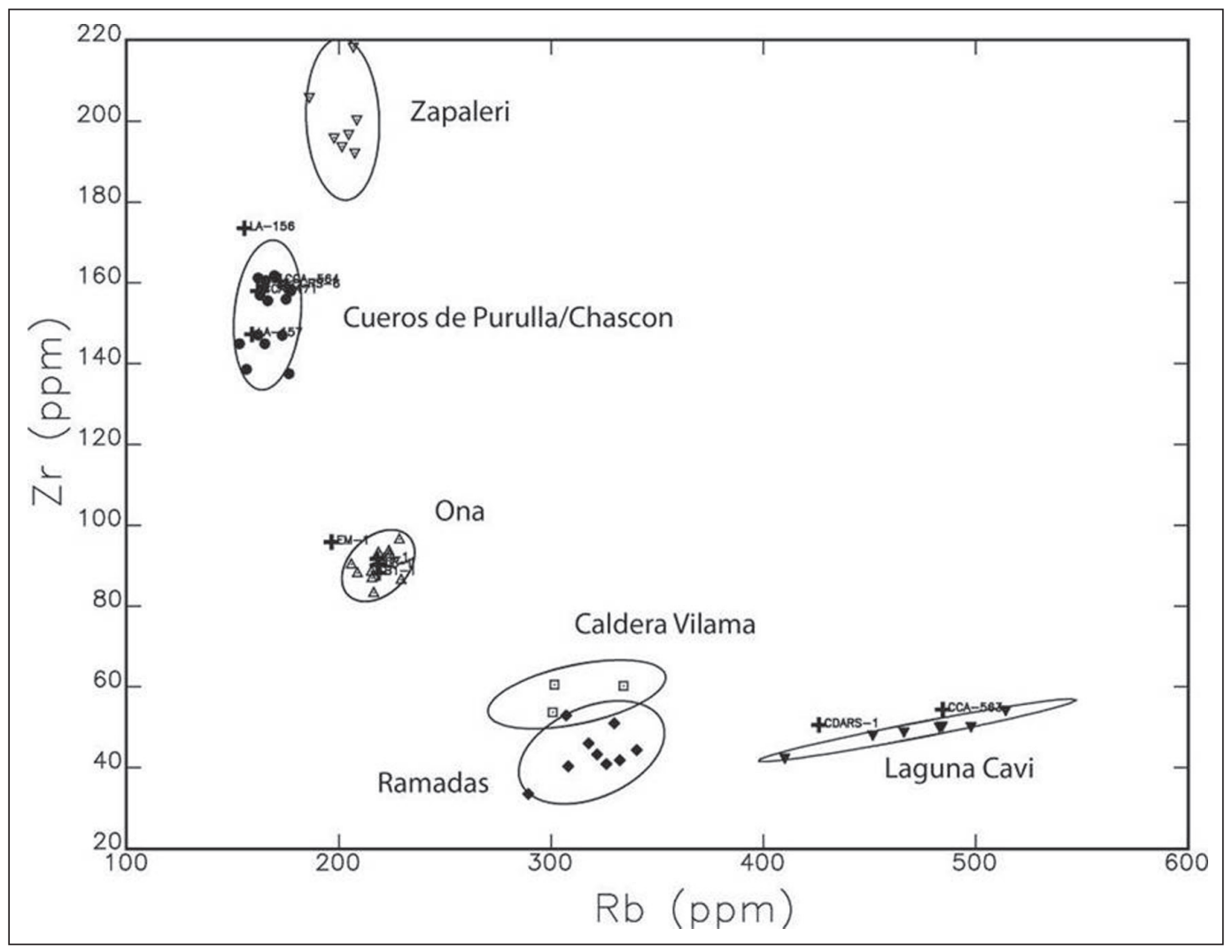

Figura 6. Diagrama bivariado de concentraciones de elementos $(\mathrm{Rb} / \mathrm{Zr})$. 


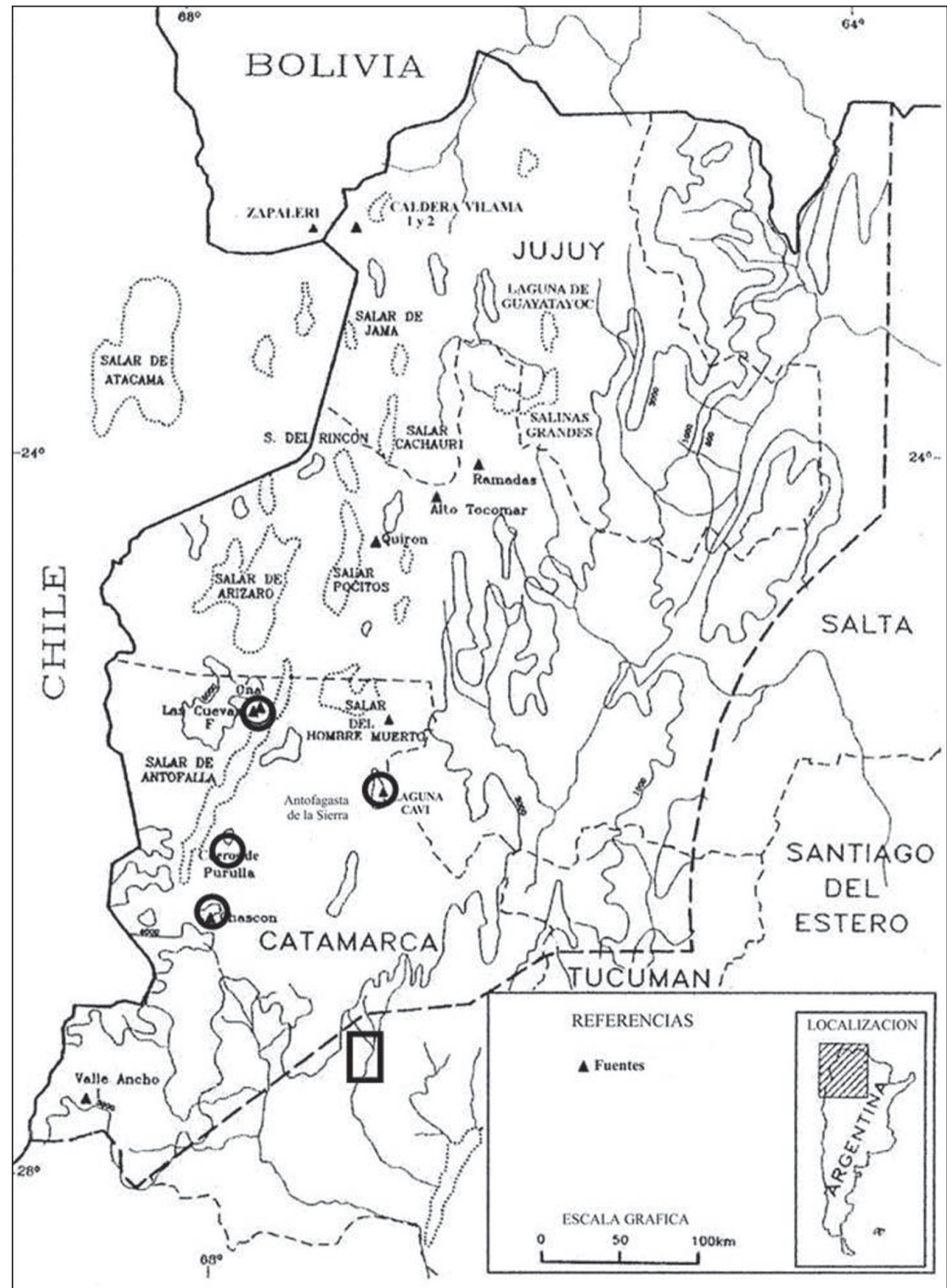

Figura 7. Fuentes de obsidianas identificadas en el valle de Hualfín (tomado y modificado de Yacobaccio et al. 2004: Fig. 1). Referencias: círculos (fuentes de aprovisionamiento de obsidianas) y rectángulo (área correspondiente al valle de Hualfín).

mayoritariamente a las variedades presentes dentro de la fuente Cueros de Purulla (Tabla 4).

Al combinar los resultados de los análisis macroscópicos y geoquímicos presentados se pudo llevar a cabo la clasificación macroscópica de todas las piezas arqueológicas de obsidiana de los diferentes sitios contemplados. Esto permitió, por un lado establecer la presencia de obsidianas procedentes de una o más fuentes en cada uno de los sitios, así como conocer su proporción (Tabla 5 y Figura 7).

\section{$*$ Discusión y CONClusiones}

La información presentada aquí, sumada a la previamente conocida para los sitios de Asampay (Flores y 
Morosi 2010), permite plantear la vinculación de las fuentes de obsidianas Ona, Cueros de Purulla, Cueros de Purulla/Chascón y Laguna Cavi con el valle de Hualfín para el período de Desarrollos Regionales/ Inka. Todas las fuentes de aprovisionamiento señaladas se encuentran en la provincia de Catamarca, dentro del sector que comprende la Puna, y a más de $100 \mathrm{~km}$ de distancia del área de estudio.

\begin{tabular}{|l|l|l|}
\hline Muestra & Análisis macroscópico & Análisis geoquímico \\
\hline LA-156 & $\mathrm{S} / \mathrm{S}$ & $\mathrm{CP} / \mathrm{CH}$ \\
\hline LA-157 & $\mathrm{CP}($ variedad 2a) & $\mathrm{CP} / \mathrm{CH}$ \\
\hline CCA-471 & $\mathrm{CP}($ variedad $2 \mathrm{~b})$ y R & $\mathrm{CP} / \mathrm{CH}$ \\
\hline CCA-563 & LC & $\mathrm{LC}$ \\
\hline CCA-564 & $\mathrm{CP}($ variedad 2a $)$ & $\mathrm{CP} / \mathrm{CH}$ \\
\hline CDARS-1 & LC & $\mathrm{LC}$ \\
\hline CCRS-6 & $\mathrm{CP}($ variedad 2a $)$ & $\mathrm{CP} / \mathrm{CH}$ \\
\hline LR-1 & $\mathrm{O}$ & $\mathrm{O}$ \\
\hline B1-1 & $\mathrm{O}$ & $\mathrm{O}$ \\
\hline LI-1 & $\mathrm{O}$ & $\mathrm{O}$ \\
\hline
\end{tabular}

Tabla 4. Comparación entre los resultados del análisis macroscópico y geoquímico de las muestras. Referencias: S/S (sin semejanza), CP (Cueros de Purulla), CP/CH (Cueros de Purulla/Chascón), LC (Laguna Cavi) y O (Ona).
Es probable que esta clase de recursos no locales hayan sido obtenidos por los pobladores del lugar a partir de algún tipo de intercambio, que implicara parentesco y/u otro tipo de vínculos, en donde seguramente habrían participado otros ítems (p. e., sal, animales, minerales, madera, entre otros). Si bien el lapso temporal considerado parece estar marcado por el conflicto generalizado, distintos investigadores señalan que las relaciones continuarían y que no habría problemas para ningún tipo de intercambio, incluido el de materias primas (Nielsen 2007b; Elías y Escola 2010).

A partir de la identificación microscópica de la obsidiana realizada por sitio fue posible observar que la presencia de las fuentes de obsidianas identificadas no es homogénea dentro de la zona de estudio. En todos los sitios se reconoció la existencia de obsidiana Ona, siendo puntualmente en los sitios Barrancas, Lajas Rojas 2 y 4 y Loma de Ichanga, la única variedad registrada hasta el momento. En los demás sitios, a saber: Loma de los Antiguos, Campo de Carrizal y Cerro Colorado se constató, además, la presencia de otras fuentes. Si bien en estos tres sitios se identificaron obsidianas de la fuente Cueros de Purulla, sólo en Loma de los Antiguos y Campo de Carrizal se hallaron especimenes correspondientes a dos fuentes, Cueros de Purulla/Chascón, por el momento no diferenciadas ni a nivel macroscópico ni geoquímico, y por último, únicamente en Campo de Carrizal se determinaron piezas procedentes de la fuente Laguna Cavi.

\begin{tabular}{|c|c|c|c|c|c|c|c|c|c|}
\hline \multirow[b]{3}{*}{ Sitios } & \multicolumn{8}{|c|}{ Fuentes de obsidiana } & \multirow[b]{3}{*}{ Total } \\
\hline & \multicolumn{2}{|c|}{ O } & \multicolumn{2}{|c|}{$\mathrm{CP}$} & \multicolumn{2}{|c|}{$\mathrm{CP} / \mathrm{CH}$} & \multicolumn{2}{|c|}{ LC } & \\
\hline & $\mathbf{N}$ & $\%$ & $\mathbf{N}$ & $\%$ & $\mathbf{N}$ & $\%$ & $\mathbf{N}$ & $\%$ & \\
\hline LA & 141 & 98 & 2 & 1 & 1 & 1 & o & o & 144 \\
\hline Caz. & 108 & 93 & 3 & 3 & 1 & 1 & 4 & 3 & 116 \\
\hline Cco. & 10 & 83 & 2 & 17 & o & o & $\mathrm{O}$ & o & 12 \\
\hline $\mathrm{LR}_{2}$ & 2 & 100 & o & o & o & o & $\mathrm{O}$ & o & 2 \\
\hline $\mathrm{LR}_{4}$ & 3 & 100 & o & o & o & o & o & o & 3 \\
\hline B & 2 & 100 & o & o & o & o & o & o & 2 \\
\hline LI & 3 & 100 & o & o & o & o & o & o & 3 \\
\hline Total & 269 & 96 & 7 & 2 & 2 & 1 & 4 & 1 & 282 \\
\hline
\end{tabular}

Tabla 5. Obsidianas de diferentes fuentes de procedencia identificadas por sitio. Referencias: LA (Loma de los Antiguos), Caz (Campo de Carrizal), Cco (Cerro Colorado), LR2 (Lajas Rojas 2), LR4 (Lajas Rojas 4), B (Barrancas) y LI (Loma de Ichanga); Fuentes: O (Ona), CP (Cueros de Purulla), CP/CH (Cueros de Purulla/Chascón) y LC (Laguna Cavi). 
Campo de Carrizal constituye hasta el momento el único sitio del valle de Hualfín, entre los analizados, que presenta la mayor diversidad en cuanto a fuentes utilizadas.

El hallazgo de obsidiana Ona en sitios emplazados en ambas márgenes del río Hualfín, ubicados en el sector noroeste y sureste del valle, y que presentan un grado de organización social diferente, evidencia tanto su amplia distribución espacial como su libre circulación y acceso. Esto mismo parece suceder con la obsidiana Cueros de Purulla, aunque presenta una distribución y alcance menor. La obsidiana Laguna Cavi, por el contrario, hasta el momento no parecería tener una gran distribución dentro del área de estudio y su presencia se limita a un solo sitio ubicado en la margen occidental del valle.

Es interesante señalar que en los sitios en donde se registró más de una fuente de obsidiana, es la procedente de Ona la que se encuentra con mayor frecuencia en los conjuntos. Esto mismo sucede en otros sitios del noroeste argentino correspondientes al lapso temporal aquí considerado (Avalos 2003, Chaparro 2009, Elías 2011, entre otros). Yacobaccio y colaboradores (2004) señalan fuentes de menor distribución dentro del noroeste argentino, las cuales habrían acompañado a otras de mayor alcance. Entre ellas, mencionan que la fuente Cueros de Purulla habría funcionado paralelamente a la fuente Ona. La mayor o menor presencia de obsidianas de diferentes fuentes de procedencia podría ser interpretada de diversas maneras, siendo el resultado, posiblemente, de múltiples factores combinados: un mayor "acceso" a la fuente, una mejor calidad del recurso para la talla, una preferencia por las características macroscópicas de esa obsidiana, como la brillantez y transparencia u otras cuestiones, que como aquellas pudieron estar más vinculadas a "lo social" que a "lo económico" (Chaparro 2009; Escola 2007). Asimismo, no se puede dejar de señalar que estas cuestiones (presencia/ausencia de determinadas fuentes y cantidad de ítems, por lo menos en la superficie de los sitios) puedan estar supeditadas también a la recolección que realizan, y posiblemente realizaron en el pasado, los lugareños (principalmente los niños) y visitantes de la zona, dado que reconocen y aprecian este tipo de materia prima.

En los sitios considerados pudieron ser identificadas varias etapas de la secuencia operativa de obsidianas de diferentes fuentes. En general, las características observadas en los conjuntos hallados permiten plantear por una parte la introducción a los sitios de núcleos y/o lascas, es decir obsidiana como materia prima para ser trabajada en ellos, aunque además podrían haberse introducido piezas ya elaboradas, y por otra parte, un tratamiento del material bastante similar, independientemente de su origen, aspecto y calidad, relacionado con un aprovechamiento intensivo y con la manufactura de puntas de proyectil (Flores 2013). Esta cuestión será abordada en un futuro trabajo.

El presente estudio constituye un aporte a la identificación de fuentes potenciales de aprovisionamiento de obsidiana utilizadas por los pobladores del valle de Hualfín (Departamento de Belén, Catamarca, Argentina) durante el período de Desarrollos Regionales/Inka. Los resultados complementan y amplían la información precedentemente obtenida sobre el tema para la zona de estudio (Flores y Morosi 2010).

Agradecimientos A la Universidad Nacional de La Plata, por proveer la infraestructura y medios para la realización de los estudios. A la Agencia Nacional de Promoción Científica y Técnica y al CONICET, instituciones que instrumentaron becas -Dra. Marina Flores- para la consecución de las investigaciones. A la Dra. Alejandra Elías, al Lic. Martín Morosi y al Dr. Federico Wynveldt por los aportes realizados. A los Evaluadores por las valiosas sugerencias.

\section{* Referencias citadas}

AVALOS, J. 2003. Sistemas de producción lítica de las sociedades tardías de la Quebrada de Humahuaca. Cuadernos de la Facultad de Humanidades y Ciencias Sociales 20: 271-290.
CHAPARRO, G. 2009. El manejo de los recursos líticos en el pasado: sociedades pre-estatales y estatales en el área Valliserrana del noroeste argentino (1000-1536 DC.). Tesis doctoral inédita. Facultad de Filosofía y Letras, Buenos Aires. 
DE FEO, M. E. y M. C. ÁLVAREZ SONCINI. 2010. Estudio tecnomorfológico y análisis de procedencia de obsidianas recuperadas en el sitio formativo Tres Cruces I (Quebrada del Toro, provincia de Salta, Argentina). En La Arqueometría en Argentina y Latinoamérica, S. Bertolino, R. Cattáneo y A. D. Izeta (Eds.), pp. 165-170. Facultad de Filosofía y Humanidades, Córdoba.

ELÍAS, A. 2008. Estrategias tecnológicas y variabilidad de los conjuntos líticos de las sociedades de los Períodos Tardío y TardíoInka en Antofagasta de la Sierra (Prov. de Catamarca, Puna Meridional Argentina) y Doncellas (Prov. de Jujuy, Puna Septentrional Argentina). Comechingonia Virtual 1: 43-72.

2011. Estrategias tecnológicas y variabilidad de los conjuntos líticos de las sociedades tardías en Antofagasta de la Sierra (Provincia de Catamarca, Puna Meridional argentina). Tesis doctoral inédita, Facultad de Filosofía y Letras, Universidad de Buenos Aires.

ELÍAS, A. y P. ESCOLA. 2010. Viejos y nuevos horizontes: obsidianas entre las sociedades agrícolas-pastoriles del Período Tardío en Antofagasta de la Sierra (provincia de Catamarca, Puna Meridional Argentina). Revista Española de Antropología Americana 40 (2):9-29.

ELÍAS, A.; D. OLIVERA, P. TCHILINGUIRIAN, M. GLASCOCK y P. ESCOLA. 2009. Procedencia de obsidianas de sitios arqueológicos tardíos y tardíos-inkas de Antofagasta de la Sierra (Prov. de Catamarca, Puna Meridional argentina) a través de florescencia de rayos x. En Arqueometría Latinoamericana: Segundo Congreso Argentino y Primero Latinoamericano, vol. 1, O. Palacios, C. Vásquez, T. Palacios y E. Cabanillas (Eds.), pp. 109-114. Comisión Nacional de Energía Atómica (CNEA). Buenos Aires.

ESCOLA, P. 2000. Tecnología lítica y sociedades agro-pastoriles tempranas. Tesis doctoral inédita, Facultad de Filosofía y Letras. Universidad de Buenos Aires.

2004. Variabilidad en la explotación y distribución de obsidianas en la Puna Meridional Argentina. Estudios Atacameños 28: 9-24.

2007. Obsidianas en contexto: tráfico de bienes, lazos sociales y algo más. En Sociedades Precolombinas Surandinas. Temporalidad, Interacción y Dinámica Cultural del NOA en el ámbito de los Andes Centro-Sur, V. Williams, B. Ventura, A. Callegari y H. Yacobaccio (Eds.), pp. 73-87. Buenos Aires. Taller Internacional de Arqueología del NOA y Andes Centro-Sur.

ESCOLA, P.; M. GLASCOCK, M. KORSTANJE y N. SENTINELLI. 2009. Laguna Cavi y El Médano: obsidianas en circulación caravanera. En Arqueometría Latinoamericana: Segundo Congreso Argentino y Primero Latinoamericano, vol. 1, O. Palacios, C. Vásquez, T. Palacios y E. Cabanillas (Eds.), pp. 103-108. Comisión Nacional de Energía Atómica (CNEA). Buenos Aires.
ESCOLA, P. y S. HOCSMAN. 2007. Procedencia de artefactos de obsidiana de contextos arqueológicos en Antofagasta de la Sierra (ca.4500-3500 AP). Comechingonia 10: 49-62.

ESCOLA, P.; A. NASTI, J. REALES y D. OLIVERA. 1992-93. Prospecciones arqueológicas en las quebradas de la margen occidental del Salar de Antofalla, Catamarca (Puna Meridional argentina): resultados preliminares. Cuadernos del Instituto $\mathrm{Na}$ cional de Antropología y Pensamiento Latinoamericano 14: 171-189.

FLORES, M. 2013. Aprovisionamiento y manejo de materias primas líticas durante el Período de Desarrollos Regionales/Inka en el valle de Hualfín (Depto. de Belén, Catamarca). Tesis Doctoral Inédita para optar por el grado de Doctor en Ciencias Naturales, Facultad de Ciencias Naturales y Museo, Universidad Nacional de La Plata.

FLORES, M. y M. MOROSI. 2010. ¿De dónde vienen?: obsidianas de la localidad de Azampay (Dto. de Belén, Catamarca). En La Arqueometría en Argentina y Latinoamérica, S. Bertolino, R. Cattáneo y A. D. Izeta (Eds.), pp. 177-182. Facultad de Filosofía y Humanidades, Córdoba.

GONZÁLEZ, A. 1998. La cultura de La Aguada. Arqueología y diseños. Filmediciones Valero. Buenos Aires.

GONZÁLEZ, A. y J. PÉREZ. 1972. Argentina Indigena. Visperas de la conquista. Paidós. Buenos Aires.

GONZÁLEZ BONORINO, F. 1950. Geologíay Petrografía de las Hojas $12 d$ (Capillitas) y 13d (Andalgalá). Ministerio de Industria y Comercio de la Nación, Dirección General de Minería. Boletín No 70 . Buenos Aires.

1972. Descripción Geológica de la Hoja 13c, Fiambalá, Provincia de Catamarca. Ministerio de Industria y Minería. Subsecretaría de Minería. Dirección Nacional de Geología y Minería. Boletín No 127. Buenos Aires.

NIELSEN, A. 2002. Asentamientos, conflicto y cambio social en el Altiplano de Lípez (Potosí). Revista Española de Antropología Americana 32: 179-205.

2007a. Armas significantes: tramas culturales, guerra y cambio social en el sur andino prehispánico. Boletín del Museo Chileno de Arte Precolombino 12(1): 9-41.

2007b. Bajo el hechizo de los emblemas: políticas corporativas y tráfico interregional en los Andes Circumpuneños. En Producción y Circulación Prehispánicas de Bienes en el Sur Andino, A. Nielsen, M. C. Rivolta, V. Seldes, M. M. Vázquez y P. Mercolli (Eds.), pp. 393-411. Brujas, Córdoba. 
NÚÑEZ REGUEIRO, V. 1974. Conceptos instrumentales y marco teórico en relación al análisis del desarrollo cultural del noroeste argentino. Revista del Instituto de Antropología. Universidad Nacional de Córdoba 5: 169-19o.

RUIZ HUIDOBRO, O. 1975. Descripción geológica de la Hoja 12 c, Laguna Helada, Provincia de Catamarca. Ministerio de Economía, Secretaria de Estado de Recursos Naturales y Ambiente Humano, Subsecretaría de Minería, Servicio Geológico Nacional. Boletín $\mathrm{N}^{\circ}$ 146. Buenos Aires.

SCATOLÍN, C. y M. LAZZARI. 1997. Tramando redes: obsidianas al oeste del Aconquija. Estudios Atacameños 14: 189-209.

SEMPÉ, M. C. 1981. Investigaciones arqueológicas en el Departamento de Belén (Catamarca). Novedades del Museo de La Plata 1 (2): 18-19.

1999. La Cultura Belén. En Actas del XII Congreso Nacional de Arqueología Argentina, Tomo III, pp. 250-258. Universidad Nacional de La Plata, La Plata.

2005. El Período Tardío en Azampay: el señorío Belén y su modelo geopolítico. En Azampay: presente y pasado de un pueblito catamarqueño, M.C. Sempé, S. Salceda y M. Mafia (Eds.), pp. 365-380. Al Margen, La Plata.
SPROVIERI, M. y L. BALDINI. 2007. Aproximación a la producción lítica en sociedades tardías. El caso de Molinos I, valle Calchaquí Central (Salta). Intersecciones en Antropología 8: 135-147.

SPROVIERI, M. y M. GLASCOCK. 2007. Aproximación a la circulación de obsidiana en el valle Calchaquí salteño entre los siglos IX y XV. En Actas del XVI Congreso de Arqueología Argentina. Número especial de Revista Sacarina, Tomo III, pp. 221-226. Facultad de Humanidades y Ciencias Sociales, Universidad Nacional de Jujuy, San Salvador de Jujuy.

TARRAGÓ, M. 2000. Chacras y pukara. Desarrollos sociales tardíos. En Los pueblos originarios y la conquista, Nueva Historia Argentina, Tomo I, M. Tarragó (Comp.), pp. 257-300. Sudamericana. Buenos Aires.

WYNVELDT, F. 2009. La Loma de los Antiguos de Azampay. Un sitio defensivo del Valle de Hualfín (Catamarca, Argentina). Sociedad Argentina de Antropología, Buenos Aires.

YACOBACCIO, H.; P. ESCOLA, F. PEREYRA, M. LAZZARI y M. GLASCOCK. 2004. Quest for ancient routes: obsidian sourcing research in Northwestern Argentina. Journal of Archaeological Science 31(2): 193-204. 